\title{
Thermal Simulation of Big Area Additive Manufacturing
}

\author{
Brian Friedrich ${ }^{1}$, Kyosung Choo ${ }^{1}$ \\ ${ }^{1}$ Mechanical and Industrial Engineering Department, Youngstown State University, \\ Youngstown, $\mathrm{OH} 44555$, United States \\ bkfriedrich@student.ysu.edu,kchoo@ysu.edu
}

\begin{abstract}
A common failure mode of Big Area Additive Manufacturing (BAAM) is the phenomena of slumping. Slumping occurs when the structure retains excessive heat, often seen when there is insufficient cooling between layers. This study developed a transient thermal simulation model to aid in predicting the slumping phenomena, specifically in overhanging features. The simulation was modeled in ANSYS where the walls were created to match the dimensions in the experimental pyramid at $12.5 \mathrm{~mm}$ wide with a thickness of 5 $\mathrm{mm}$. The structures overall size was $1.06 \mathrm{~m}$ by $0.77 \mathrm{~m}$ and 25 layers tall. Each layer was created independently to allow for element birth/death commands and for individual layer mesh parameters. Using the built-in element birth/death commands each layer would be inserted on top of the previous layer. As each new layer is activated a temperature input of $202^{\circ} \mathrm{C}$ is applied then subsequently turned off as the next layer is activated. The printing material, ABS (Acrylonitrile Butadiene Styrene), properties and heat transfer coefficient of the structures are functions of temperature. The simulation model is compared to an experimentally measured part. A FLIR E60 thermal imaging camera is utilized to capture the vertical thermographic profile of the build. The camera was paired with a computer running the FLIR Tools software package in order to record, save, and later analyze the thermographic history. The thermal images also captured three different vertical lines traversing all layers. Each pixel in the lines would record the corresponding temperatures of the structure. The data taken from the three lines show that the cooling present in the structure is of an exponential form. This result matches what was produced from the simulation, within $5 \%$ error. The simulation allows for dwell times to be adjusted in the model until failure is no longer predicted. Utilizing these transient thermal modeling techniques will aid BAAM designers to identify potential slumping during the print process.
\end{abstract}

Keywords: Thermal modeling; Process monitoring; Simulation

\section{Introduction}

Big Area Additive Manufacturing (BAAM) advances the benefits of general additive manufacturing to include large complex geometries, the elimination of hard tooling, and the paradigm of direct digital manufacturing. During production of the experimental parts, all the structures failed when there was insufficient solidification of a layer before the subsequent layer was added. The design of the experimental structure allows for each layer to be produced at incrementally quicker print times. The quicker print times do not allow for each layer to effectively cool to prevent slumping beyond the plane of displacement threshold. Figure 1 shows the pyramid printed almost to completion before failure occurred.

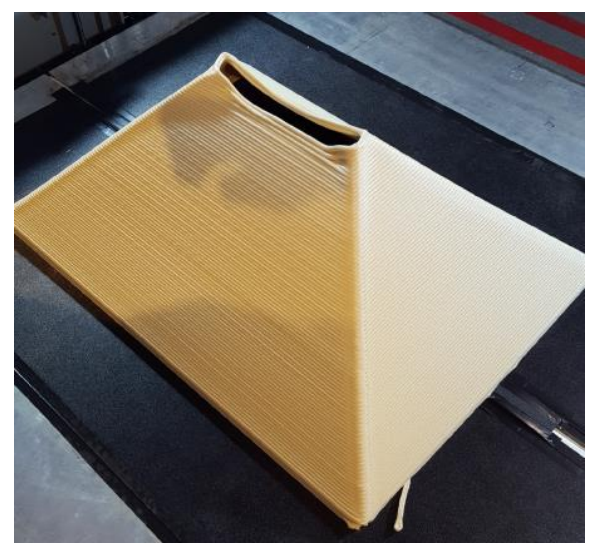

Fig. 1: The fabricated structure [5]. 
The figure shows structural failure at the top where enough heat is retained in the model to initiate slumping. The temperature recorded near the failed region was above the glass transition temperature when failure occurred. To prevent this failure the temperature is required to be below the glass transition temperature which will serve as support for subsequent layers. Thermal monitoring and modeling in polymer extrusion 3D printing have both been explored in order to balance the trade-off between maintaining sufficient heat for bonding between layers versus solidifying one layer sufficiently before depositing the next. Infrared imaging has been explored as a quality measurement tool for thermal monitoring during additive manufacturing, but remains largely absent in production systems [1-5]. This work demonstrates the clear value of tightly controlling the temperature envelope and geometry. For effective printing the temperature should remain between two temperature limits. The low temperature limit should cause sufficient solidification in which overhanging features do not deflect unintentionally, and the high temperature limit should be warm enough that layers still bond well. Therefore, it is important to understand the thermal characteristics for the BAAM system in order to enhance the stability of the BAAM part and decrease the failure factor of the printing systems.

\section{Simulation Model}

\subsection{Structure}

The model for ABS (Acrylonitrile Butadiene Styrene) experimentally created in Choo et al. [5] is shown in Figure 2. The model was designed for failure to occur which is seen in Figure 1. The bead width used in this experiment is $12.5 \mathrm{~mm}$ while the thickness is $5 \mathrm{~mm}$, these are typical sizes for creating BAAM models. The geometry used in this experimental structure allows for different cooling rates to be studied by having long and short sides of the structure.

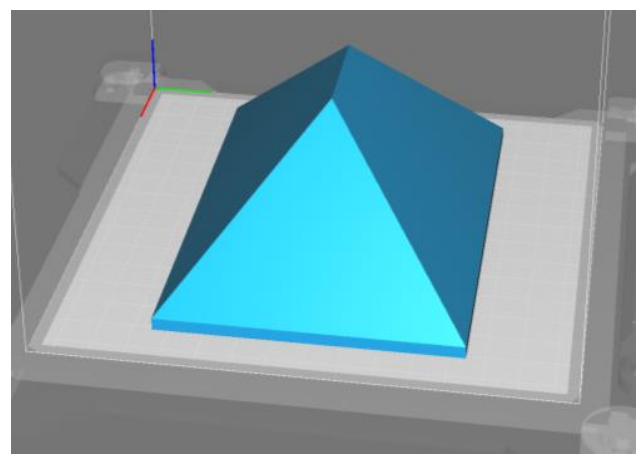

Fig. 2: Benchmark structure used in the experimentation and modeling with ABS (Acrylonitrile Butadiene Styrene) [5].

\subsection{Simulation Model}

The dynamic simulation was modeled in ANSYS where the walls were created to match the dimensions in the experimental pyramid at $12.5 \mathrm{~mm}$ wide with a thickness of $5 \mathrm{~mm}$. The structures overall size was $1.06 \mathrm{~m}$ by $0.77 \mathrm{~m}$ and 25 layers tall. The structural steel floor print plate was created to provide a constant base temperature during the simulation. The ambient temperature of the model was set at $22^{\circ} \mathrm{C}$. Each layer was created independently to allow for element birth/death commands and for discrete mesh parameters. The ABS (Acrylonitrile Butadiene Styrene) material properties and heat transfer coefficient of the structures were input as a function of temperature.

Using the built-in element birth/death commands each layer was inserted on top of the previous layer. As each new layer is activated a temperature input of $202^{\circ} \mathrm{C}$ is applied then subsequently turned off as the next layer is activated. 


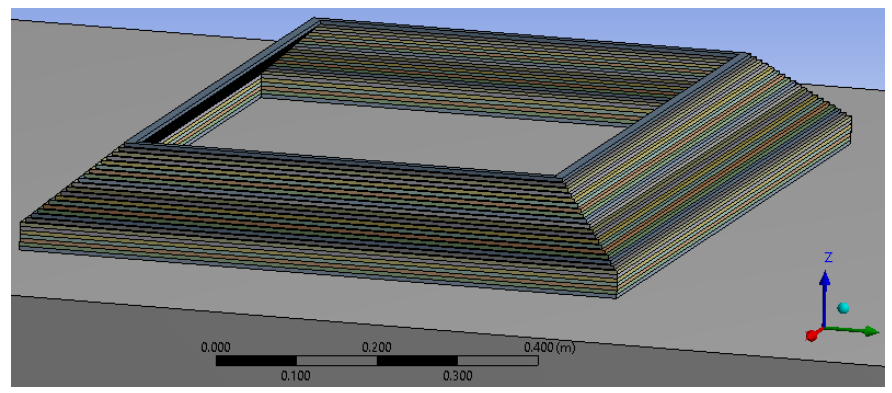

Fig. 3: Simulation model geometry.

The mesh created for each layer and floor used the same settings. The resolution, defeature size, element order, element size, and element quality were all set to default and program controlled. This created a total of 24210 nodes and 2434 elements. Figure 4 shows the details of the mesh and node locations of the floor and layers.

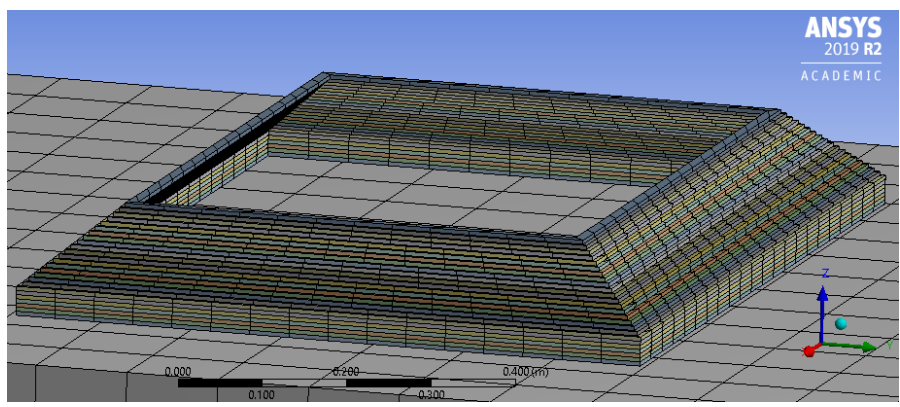

Fig. 4: Mesh distribution.

\subsection{Experiment}

Two different devices were used to record the experimental portion of the build. First, for documentation purposes, a Nest Drop Cam was used for a visual recording of the build. The Nest camera recording also allowed the verification of the printing layer time for any given layer in the build. The second method of recording utilized a FLIR E60 thermal imaging camera to capture the vertical thermographic profile of the build. The camera was paired with a computer running the FLIR Tools software package in order to record, save, and later analyze the thermographic history. Figure 5 shows the location of the monitoring during the printing. After the fabrication, in order to measure the in-plane deflection of the experimental trials, each piece was laser scanned using a 9 foot Faro Platinum portable CMM arm equipped with a Faro Blue Line laser scanner. The scan data was captured and analyzed using Polyworks Inspector 2017 software [5].

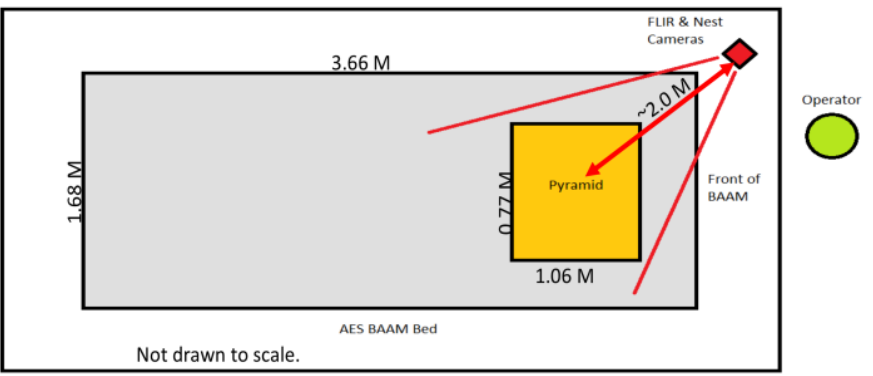

Fig. 5: Schematic representation of the location of the printing and thermal recording devices [5]. 


\section{Results and Discussion}

Figure 6 shows the thermography image for the build in mid-fabrication, approximately 25 layers [5]. Emissivity settings used in the thermography was 0.87 . It can easily be seen that the temperature decreases monotonically from the top layer until it reaches the constant temperature base plate.

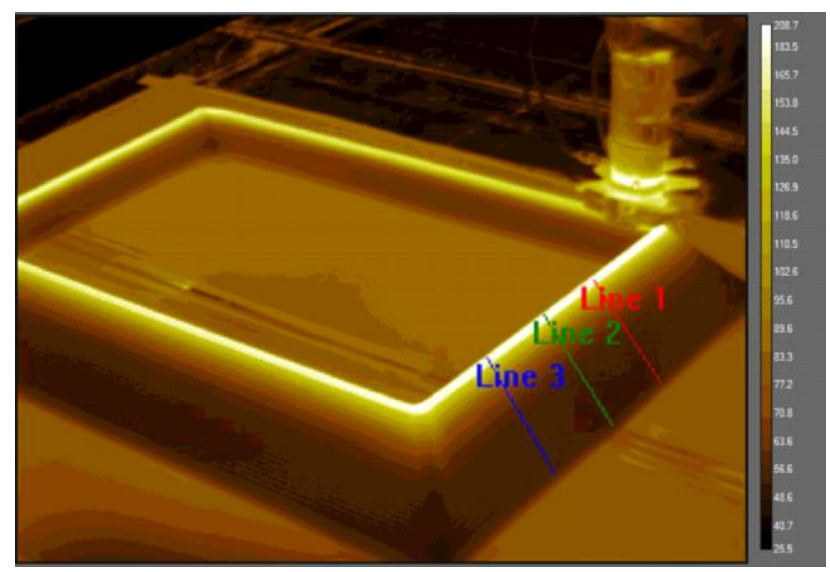

Fig. 6: Infrared imaging of the fabrication with three thermal vertical profiles in Celsius [5].

The results from the thermal simulation are shown in Figures 7 and 8 . The temperature varies from $198.7^{\circ} \mathrm{C}-50^{\circ} \mathrm{C}$ from the top layer to the bottom layer.

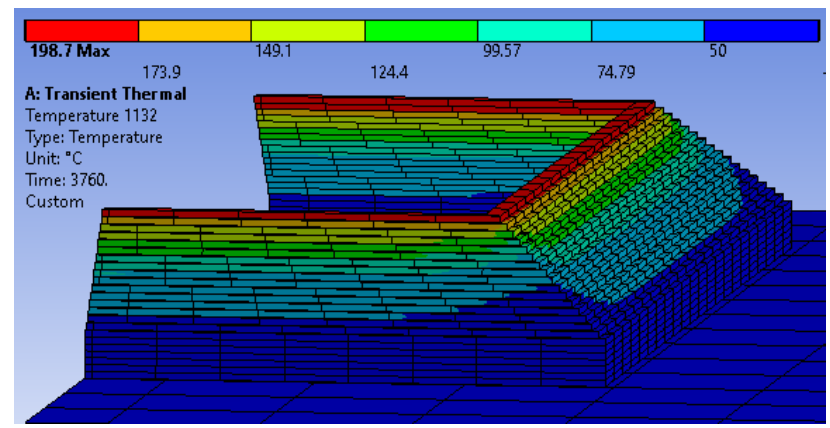

Fig. 7: Temperature profile of the printing layers.

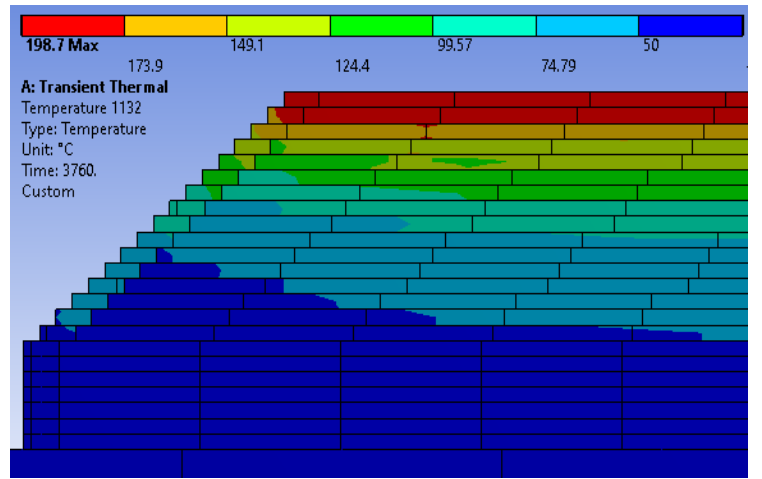

Fig. 8: Temperature profile of the cross section for the printing layers.

Figure 9 shows a comparison to the print layer and the corresponding temperature after 25 layers have been printed. This plot illustrates that the simulation was able to accurately, within $5 \%$ error, predict the temperature profile when compared to the experimental model. 


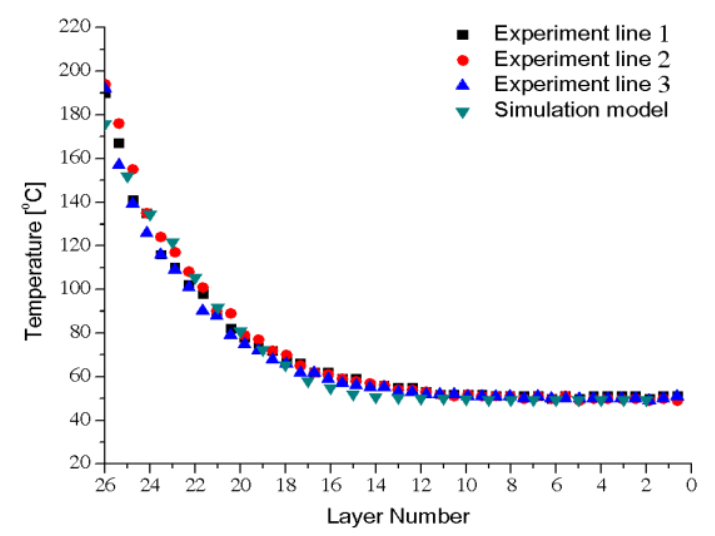

Fig. 9: Comparison of the thermal model to the three lines identified in Figure 6.

From Figure 6 it can be seen that the thermal images also captured three different vertical lines traversing all layers. Each pixel in the lines recorded the corresponding temperature of the structure. The data taken from the three lines show that the cooling present in the structure is of an exponential form. Since the design of the structure utilizes overhanging features and the solidification occurs exponentially it is very susceptible to slumping as more layers are added on at quicker rates. Utilizing these modeling techniques will aid BAAM designers to identify potential slumping. Specifically slumping dependent on wall thickness, overhanging geometry, and high temperature retention.

The results herein show that a smaller thermal mass is needed when printing higher overhanging features. It can also be inferred that the exponential cooling is ideal for the printing process. As the print becomes taller it is important for the base layers to have solidified which allow for overhanging features to be restrained. The lower setting temperature also allows for minimal material displacement during the print which allows for higher efficiency printing.

\section{Conclusion}

This study produced a simulation model to predict the temperature profile of printed parts in terms of print layers. The model was created to account for conduction, convection, and radiation. Results from the simulation measure inside of a $5 \%$ error tolerance to the experimental data. The printed parts were monitored with an infrared camera during fabrication to record the thermal profiles throughout printing, and afterwards, scanned with a 3D scanner to assess the geometrical deviations from the original design. The results showed that the heat loss during the printing process by layers is exponential. This suggests that (1) when the print is near failure the previous layer has not sufficiently cooled to offer enough structural support for the next bead and (2) when the print is not near failure, exponential cooling is preferred since the lower setting temperature gives the print better structural performance when overhanging features are being printed.

\section{References}

[1] Brenken, B., Barocio, E., Favaloro, A, Kunc, V., and Pipes, R.B., 2018, "Fused Filament Fabrication of Fiber-Reinforced Polymers: A Review." Additive Manufacturing, 21 pp. 1-16.

[2] Gibson, I, Rosen, D., and Stucker, B., 2015, “Additive Manufacturing Technologies”, 3D Printing, Rapid Prototyping, and Direct Digital Manufacturing. Springer.

[3] Kim, C., Espalin, D., Cuaron, A., Perez, M.A., MacDonald, E., and Wicker, R. B., 2015, “A Study to Detect a Material Deposition Status in Fused Deposition Modeling Technology." IEEE International Conference on Advanced Intelligent Mechatronics (AIM), pp. 779-83.

[4] Kousiatza, Charoula, and Karalekas, D., 2016, "In-Situ Monitoring of Strain and Temperature Distributions during Fused Deposition Modeling Process." Materials \& Design, 97, pp. 400-406.

[5] Choo, K., B. Friedrich, T. Daugherty, A. Schmidt, C. Patterson, M. Abraham, B. Conner, K. Rogers, E. MacDonald, P. Cortes, 2019, "Heat Retention Modeling of Big Area Additive Manufacturing," Additive Manufacturing, 28, pp. 325332. 